\title{
Malvinas en las escuelas. La
} construcción de la cuestión Malvinas en el sistema educativo de nivel medio en el Gran Río Cuarto

Malvinas in schools. The construction of the Malvinas question in the middle level educational system in the Great Río Cuarto

Gustavo Eduardo García. ${ }^{1}$

\section{Resumen}

El presente trabajo tiene como objetivo analizar a las prácticas socio-comunitarias como experiencia de extensión, implementadas por la Universidad Nacional de Río Cuarto en 2009. A la vez se intentará explicitar de qué manera la misma ha sido ejecutada y cuales han sido los resultados obtenidos a la hora de la generación de conciencia sobre la cuestión Malvinas, tanto entre los estudiantes universitarios que participaron de las mismas, para los estudiantes, docentes y directivos de las escuelas del Gran Río Cuarto en las que las mismas se llevaron a cabo y los miembros de la agrupación de veteranos de guerra con quienes se trabajó durante todo el proceso.

Palabras clave: Prácticas socio comunitarias, Escuelas de nivel medio, Agrupación de Veteranos de Guerra, Cuestión Malvinas

\section{Abstract}

The objective of this work is to analyze socio-community practices as extension practices, implemented by the National University of Río Cuarto in 2009. At the same time, an attempt will be made to explain how it has been carried out and what the results have been. at the time of raising awareness about the Malvinas issue, both among the university students who participated in them, for the students, teachers and managers of the schools in which they were held and the members of the group of war veterans with whom they worked throughout the process.

Recibido: 30 de abril de 2020 Aceptado: 3 de julio de 2020 Publicado: 10 de julio de 2020

${ }^{1}$ El autor es Abogado y Licenciado en Ciencia Política (UNRC), doctorando en Relaciones Internacionales (UNR), Prof. Adjunto en cátedras de Derecho Internacional Público (UNSL), Derecho Internacional Privado (UNRC) y Seminario de Integración Regional (UNRC), además es Director (2019 - 2020) del Ateneo de Estudios Internacionales (AEI) de la Universidad Nacional de Río Cuarto donde coordina el Área Malvinas desde de 2012. Correo electrónico: gustavo.garcia.arg@gmail.com 
Keywords: Socio-community practices, Middle level schools, War Veterans Association, Malvinas Question

\section{Introducción}

Desde el año 2009, la Universidad Nacional de Río Cuarto incorporó, a través de la Resolución $322 / 2009^{2}$ la de denominadas prácticas socio comunitarias en todas las carreras de la institución. Las mismas pueden incluirse en las currículas como módulos dentro de las asignaturas, seminarios, talleres o espacios de prácticas profesionales, según consideren los docentes a cargo. ${ }^{3}$

La finalidad que se propusieron las mismas, fue la de dar sentido y generar compromiso social en todas aquellas personas que forman parte de la comunidad universitaria, a la vez que promueve la formación socio-crítica y ciudadana de los futuros egresados. En su ejecución, los estudiantes deben aplicar los contenidos teóricos vistos en las diferentes materias de la carrera, sea en asignaturas, seminario, talleres o espacios de prácticas profesionales, a través de la realización de proyectos que contribuyen a la comprensión y resolución de problemas sociales, medioambientales y/o económicos. Todo bajo la dirección del docente a cargo.

Entre los ejes prioritarios de las prácticas socio-comunitarias tenemos temas como salud, violencia, mundo del trabajo, economía social, medio ambiente y educación. En este último eje se introduce la iniciativa que, a través del presente trabajo se pretende explicitar.

La introducción de la cuestión Malvinas en las escuelas de nivel medio ha sido considerada como una materia pendiente dentro de las currículas escolares, que si bien muchas veces está dentro de los contenidos mínimos, no recibe, por diferentes factores, la atención que podría darse a partir de la interacción con instituciones como las universidades, donde el tema es estudiado desde diferentes disciplinas, especialmente el Derecho, la Ciencia Política o las Relaciones Internacionales. De este modo, la práctica socio-comunitaria se ha constituido en la respuesta tan esperada tanto por docentes y autoridades de las escuelas del nivel medio como de la agrupación de veteranos de guerra de la ciudad para poder instalar cada año la discusión sobre la Guerra de Malvinas, pero por sobre todas las cosas, los

\footnotetext{
${ }^{2}$ La misma puede consultarse en el sitio web de la UNRC, en la sección de la Secretaria de Planificación y Relaciones Institucionales: https:/www.unrc.edu.ar/unrc/planeamiento/docs/res322-09-creacionpsc.pdf

${ }^{3}$ La UNRC fue la primera universidad nacional que institucionaliza esta experiencia, lo que fue reconocido con el Primer Premio Presidencial "Prácticas Educativas Solidarias en Educación Superior 2010”. Le han seguido en esta iniciativa, con diversas modalidades, otras casas de altos estudios como la Universidad Nacional de Buenos Aires, Universidad Nacional de Mar del Plata, Universidad Nacional General Sarmiento, Universidad Nacional de Avellaneda, Universidad Nacional de Rosario.
} 
fundamentos jurídicos, políticos e históricos sobre los que descansa la posición argentina a nivel internacional sobre los territorios irredentos.

\section{Fundamentos teóricos y metodologías}

La Resolución 322 del año 2009 en su anexo detalla cuales son los fundamentos de las prácticas socio-comunitarias, poniendo el énfasis en la concepción política de la universidad pública como actora y formadora en y para el compromiso social, político y cultural de toda la comunidad universitaria.

Seguidamente establece que encuentra su justificación en tres ejes:

* Antecedentes y evaluaciones institucionales

El antecedente más importante lo constituyen las Primeras Jornadas de Prácticas Socio-comunitarias Solidarias en la Educación Superior, desarrolladas en la Universidad Nacional de Río Cuarto en 2009, organizadas por la Secretaría de Planificación y Relaciones Institucionales como actividad dentro del Proyecto de Innovación e Investigación para el Mejoramiento Estratégico Institucional (PIIMEI) denominado "Incorporación de prácticas socio-comunitarias al currículo". En ellas se pudo visualizar, a partir de los trabajos de extensión, voluntariado y afines desarrollados por estudiantes y docentes desde diversas asignaturas o espacios institucionales de nuestra universidad, el interés por las iniciativas dirigidas a una educación solidaria. Se pudo conocer la existencia de experiencias de aprendizaje a partir de la inserción en organizaciones barriales, comunidades aborígenes, etc. También se pudo resaltar la exigencia desde diferentes carreras, de que este tipo de actividades se incorporen de manera obligatoria en los planes de estudio y el beneplácito de los estudiantes de seguir llevando adelante las mismas.

\section{* El marco normativo-proyectivo institucional}

La resolución expone que este tipo de proyectos posibilitaría el cumplimiento del ethos de una universidad comprometida con la transformación de su medio explicitado en el Estatuto y en el Plan Estratégico Institucional (PEI) de la UNRC. El Estatuto define a la Universidad como "un bien social, que debe contribuir a la definición y a la resolución de problemas sociales de los grupos o sectores más vulnerables, al desarrollo económico nacional, a la preservación del medio ambiente y a la creación de una cultura y una conciencia nacional y solidaria”. El PEI, por su parte establece como lineamiento estratégico la pertinencia social y solidaria de la universidad entendida como producción, enseñanza y utilización de conocimientos científicos y tecnológicos para contribuir a pensar y resolver los problemas críticos de la región así como a la valoración de la dimensión socio-crítica en la formación de los estudiantes que les posibilite el estudio, análisis e intervención en problemáticas sociales, políticas y culturales. Se propone, además la construcción de un currículo 
"que integre: formación general, profesional específica, científico-técnica y sociohumanística; que coadyuve a la creación de conciencia social y ciudadana, en el marco de una función crítica de la Universidad” (PEI-UNRC).

\section{* Bases conceptuales asumidas como marco teórico referencial}

El proyecto se sustenta en particulares concepciones de aprendizaje, de conocimiento y de relación entre la Universidad y la sociedad. Supone una concepción de aprendizaje situado o contextualizado que asume la relación entre conocer, hacer y contexto como inseparables o de mutua constitución. Esto supone recuperar el concepto de competencias concebidas como saberes en acción o como praxis que articula conocimiento y práctica en contexto. Adaptando el concepto que construimos conjuntamente en esta Universidad sobre competencias y ajustándolo al contexto de prácticas socio-comunitarias decimos que en estas situaciones los estudiantes construyen capacidades complejas y potenciales para actuar en contextos comunitarios reales, integrando y usando conocimientos y procedimientos de las disciplinas y actitudes o valores solidarios, de manera estratégica y con conciencia ética y social. Se trata de saberes más que de conocimientos ya que el saber no se reduce a la dimensión conceptual o declarativa del conocimiento sino que integra sus dimensiones procedimentales, actitudinales, valorativas y éticas. Por otro lado, son saberes en acción ya que se construyen en contextos de acción y para la acción.

El conocimiento se concibe como complejo, distribuido, generado a partir de problemas socialmente relevantes y orientados a su resolución. Ello interpela a la gestión social del conocimiento que promueva la producción, uso y distribución del mismo con fines de transformación socio-económica-ambiental.

La relación entre Universidad y sociedad se entiende como de diálogo, comunicación, cooperación y aprendizaje conjunto. En estos módulos se conjugarían diversos saberes: académicos, científicos, humanísticos, técnicos, cotidianos, prácticos, populares, etc. Por ello la propuesta responde a lo que Sousa Santos ha denominado “ecología de saberes". Se trata de un conjunto de prácticas que promueven un diálogo y convivencia entre diversos saberes que pueden enriquecerse en la interacción. Requieren la conformación de comunidades de aprendizaje y epistémicas amplias o redes de ciudadanos, trabajadores, profesionales, profesores, investigadores, estudiantes, organizaciones de la sociedad civil y del Estado, que comparten y construyen un conocimiento común en situaciones de horizontalidad.

Por ello, el objetivo general de las PSC es el de construir y afianzar un currículo que coadyuve a la creación de conciencia social y ciudadana, en el marco de una función crítica de la Universidad. Para ello, se propone como objetivos específicos: 1) Desarrollar actitudes de solidaridad, sensibilidad y compromiso social en los estudiantes, articuladas con el aprendizaje de contenidos de sus respectivos campos 
profesionales y científicos, a través de su interacción con problemas de la realidad social. 2) Contribuir a través de la enseñanza, la investigación y el servicio a la resolución de problemas sociales críticos y 3) Enriquecer la actividad académicocientífica de la Universidad con su interacción y comunicación con ámbitos de la realidad social. ${ }^{4}$

\section{Organización y gestión de la Extensión}

En principio y teniendo en cuenta lo establecido por la Res. 322/2009 antes mencionada, la programación e implementación de las prácticas socio-comunitarias estará a cargo de las Comisiones Curriculares Permanentes o Departamentos según corresponda. Las Facultades fijarán las prioridades institucionales en el marco de este proyecto, reglamentarán su implementación y arbitrarán los medios para la coordinación institucional de la programación curricular de las prácticas sociocomunitarias. $^{5}$

La propuesta de presentar proyectos de este tipo baja a las Facultades a través de la Secretaría de Planeamiento y Relaciones Institucionales que depende de Rectorado de nuestra universidad. De esta manera, tanto las autoridades de la Facultad de Ciencias Humanas como las de la mencionada secretaría vienen trabajando en conjunto para estimular a los diferentes departamentos que conforman la Facultad a los fines de que los docentes de las diferentes carreras incorporen prácticas sociocomunitarias en el dictado de las materias. La idea en el corto plazo es incorporarlas al currículo de todas las carreras de la Universidad Nacional de Río Cuarto, lo que haría que su ejecución sea obligatoria, y a mediano y largo plazo, que las prácticas se institucionalicen, es decir, que pasen a ser ejecutadas por la propia Facultad, independientemente de los docentes que las presentaron. Esto como una manera de darle continuidad a las mismas y teniendo en cuenta la importancia que la actividad de extensión ha tenido y sigue teniendo para la comunidad.

La práctica socio-comunitario que analizaremos, se llevó a cabo a partir de tres carreras que forman parte del Departamento de Ciencias Jurídicas, Políticas y Sociales de la Facultad de Ciencias Humanas. Me refiero a las carreras de Abogacía,

\footnotetext{
${ }^{4}$ Resol. 322/2009 antes citada.

${ }^{5}$ Resulta de interés destacar la existencia de un Consejo Social Universitario, dependiente de Rectorado de la UNRC, quien se encarga de realizar de forma periódica un relevamiento sobre cuáles son las necesidades de las diferentes organizaciones e instituciones de la ciudad y de la región, lo que permite conocer los requerimientos concretos que después se intentarán coordinar a través de las diferentes Facultades a los fines de que desde las carreras puedan presentarse proyectos de prácticas socio comunitarias que satisfagan dichas necesidades concretas. $\quad$ El informe 2019 puede consultarse https://www.unrc.edu.ar/unrc/planeamiento/docs/psc/2019/infconsejosocial_Ampliaci\%C3\%B3n\%202019.pdf. (última visita, 28 de abril de 2020)
} 
Licenciatura en Ciencia Política y Profesorado en Ciencias Jurídicas, Políticas y Sociales. Las asignaturas que participaron son: Derecho Internacional Público, Historia Argentina II y Teoría de las Relaciones Internacionales.

Al encontrarnos en la primera etapa de implementación, denominada de transición, la práctica socio-comunitaria que desarrollaremos se ha incorporado a las asignaturas mencionadas bajo la modalidad de módulos. Sin embargo, al ser ejecutada la misma no solo por los docentes responsables de las asignaturas sino también por los docentes y graduados que son miembros de un centro de estudios, los resultados de la práctica no se han limitado a la actividad misma sino que ha servido de base para consolidar grupos de docentes, estudiantes y graduados que a la vez que enriquecen la mecánica misma de las prácticas, van generando nuevos espacios de interés en la temática abordada como lo es la cuestión Malvinas. Esto redunda en la conformación de grupos de estudio, proyectos de investigación, participación en eventos académicos y de divulgación en escuelas y centro de veteranos de la ciudad y la zona.

De esta manera, las prácticas constituyen el punto de partida para otra serie de actividades que buscan generar compromisos entre estudiantes, docentes y graduados, los que al vincularse con la sociedad aprenden actitudes solidarias a la vez que se forman en los contenidos de las disciplinas y aprenden competencias propias de sus respectivas prácticas profesionales que le faciliten una futura inserción laboral autónoma, crítica y solidaria.

Siguiendo el plan de acción que sugiere la normativa, las autoridades de la Facultad de Ciencias Humanas, relevaron ciertas prácticas de extensión que se venían desarrollando en el centro de estudios Ateneo de Estudios Internacionales (AEI) que depende de la misma Facultad y que nuclea a docentes de diferentes materia de las tres carreras antes mencionadas del Departamento de Ciencias Jurídicas, Políticas y Sociales. Dichas actividades se venían realizando de manera continuada desde el año 2012 y respondían a necesidades concretas de diferentes sectores de la comunidad.

Por ello, teniendo en cuenta que existía un grupo consolidado de trabajo, se consideró viable proponer la implementación de prácticas socio-comunitarias que permitieran a los estudiantes de las materias dictadas por estos docentes participar de las actividades de extensión que estos docentes estaban llevando adelante.

De esta manera, en la Convocatoria del año 2019 se presentó el proyecto de práctica socio-comunitaria "Malvinas en las escuelas. La construcción de la cuestión Malvinas en el sistema educativo de nivel medio en el Gran Río Cuarto”. Posteriormente se firmaron los acuerdos institucionales con la Agrupación de Veteranos de Guerra de la ciudad de Río Cuarto “Operativo Virgen del Rosario”, institución con quien se llevó adelante la actividad. 


\section{Práctica Socio Comunitaria y Malvinización}

La idea central de la experiencia fue poder acercar a docentes y estudiantes de nuestra universidad a una de las necesidades más importantes que hemos podido relevar con el correr de los años, como lo es la de explicar los presupuestos jurídicos, políticos e históricos en los que se funda el reclamo de soberanía de la República Argentina sobre las Islas Malvinas. En este marco, se buscó el acercamiento de los estudiantes con docentes de escuelas de nivel medio de diferentes partes del Gran Río Cuarto, donde concurren estudiantes de diferentes realidades socios económicos. A su vez, permitió que los estudiantes conocieran muchas de las realidades que los veteranos de guerra han vivido y viven en la actualidad.

\section{* Problema o demanda que origina la experiencia}

El Ateneo de Estudios Internacionales (AEI), a través de su Área Malvinas viene trabajando desde el año 2012 con la Agrupación de Veteranos de la ciudad y ha podido receptar diferentes demandas por parte de esta organización a la hora de tener que acudir a las escuelas del nivel medio en las que se les solicita que concurran a los fines de explicar a los docentes y estudiantes, diferentes temas referidos a la guerra de 1982. Sin embargo, nos han hecho llegar diferentes inquietudes a la hora de dar dichas exposiciones debido a que no poseen el conocimiento técnico-jurídico necesario para poder dar las charlas, específicamente en lo relacionado con los derechos que la República Argentina tiene sobre las islas y que son el origen de una disputa bilateral que enfrenta a nuestro país con el Reino Unido de Gran Bretaña e Irlanda del Norte. A su vez, esto también puso en evidencia que los docentes a cargo de las materias en las escuelas no tienen un conocimiento acabado sobre la temática, razón que genera una necesidad constante- cada dos de abril-, por parte de las instituciones educativas, de acudir a la agrupación de veteranos y a través de ellos a nuestro centro de estudios. Por esta razón, en los ocho años que ya han transcurrido desde que el Área Malvinas se creó y después de dar más de 20 charlas en diferentes ámbitos, vemos que la necesidad está lejos de ser satisfecha. Esto tiene como lógica consecuencia que se nos invite con mayor asiduidad cada año para participar de charlas o seminarios, no solo en la ciudad, sino también en varias localidades del sur cordobés.

\section{* Objetivos de la Prácticas Socio-comunitaria}

Objetivos generales

- Colaborar con la agrupación de veteranos de la ciudad para el desarrollo de charlas requeridas desde diferentes instituciones educativas del Gran Río Cuarto.

- $\quad$ Fortalecer el conocimiento de los docentes y estudiantes sobre los legítimos derechos argentinos sobre las Islas Malvinas.

Objetivos específicos: 
- Capacitar a estudiantes de nuestra universidad a los fines de que puedan acompañar a los veteranos de guerra de la Agrupación de Veteranos de la ciudad a las escuelas de nivel medio a los fines de poder enriquecer sus conocimientos sobre la Cuestión Malvinas.

- $\quad$ Establecer un vínculo más estrecho con la Agrupación de Veteranos de Guerra "Operativo Virgen del Rosario", que permita instalar un espacio permanente de formación y capacitación en el Gran Río Cuarto.

- Consolidar un grupo de estudio abocado al análisis de los temas vinculados a la Cuestión Malvinas en nuestra universidad a los fines de poder avanzar en los años venideros en proyectos de extensión más ambiciosos pero necesarios.

\section{* Relato de las acciones realizadas}

Las acciones realizadas fueron las siguientes:

En primer lugar, se convocó a los estudiantes interesados en participar de la práctica. Ello se llevó a cabo a partir de los docentes responsables de cada una de las asignaturas, quienes previamente explicaron previamente a los estudiantes sobre el objetivo de la actividad y la forma en que la misma se llevaría adelante. A partir de allí, los interesados se anotaron en una grilla facilitada por el docente. La coordinación con las tres cátedras fue realizada por el director del Centro de Estudios Ateneo de Estudios Internacionales (AEI).

Como segunda etapa se dictó a los estudiantes participantes, a través de la modalidad de curso extracurricular, una capacitación teórico práctica a los fines de dejar en claro cuáles eran los contenidos que se debían impartir en las escuelas. Esta instancia se consideró necesaria dado que a pesar de recibir los estudiantes los contenidos teóricos en las diferentes materias participantes, resultaba vital coordinar de manera conjunta los lineamientos más importantes. Al mismo tiempo, se pensó en hacer hincapié en las herramientas pedagógicas necesarias para estudiantes que no tenían la costumbre de estar hablando frente a alumnos de escuelas de nivel medio. Para ello, una profesora miembro del grupo de estudios, realizó una serie de recomendaciones relacionadas a la forma en que los conocimientos deberían ser impartidos para poder tener una mejor llegada a los estudiantes de las escuelas.

Dentro de esta misma etapa y como una forma de que los estudiantes participantes adquirieran un mejor conocimiento de las discusiones que se han dado en torno a la cuestión Malvinas, vinculadas con los derechos argentinos y con el desarrollo mismo del conflicto bélico, se proyectaron dos películas, una de carácter documental y otra de ficción relacionada con el drama de los veteranos al concluir la guerra.

Como tercera etapa, se realizó un encuentro entre los estudiantes que iban a participar de la actividad y los miembros de la agrupación de veteranos que iban a concurrir a las escuelas. La idea fue que los estudiantes pudiesen conocer de primera 
mano a los veteranos y sus necesidades. Ello permitió generar confianza y a la vez conocer muchas de las realidades que los veteranos han vivenciado después de la guerra y también los inconvenientes que han tenido a la hora de tener que ir a exponer a las escuelas.

Como cuarta etapa, se procedió a tomar contacto con las diferentes escuelas que participarían de las prácticas. Es importante destacar que se buscaron escuelas del Gran Río Cuarto, el que está conformado no solo por la ciudad de Río Cuarto, sino también por las localidades de Santa Catalina (Estación Holmberg) y Las Higueras. Se privilegiaron escuelas que llevaran nombre de caídos en combate. De esta manera se pudieron confirmar cuatro escuelas, dos de la ciudad de Río Cuarto, una de Santa Catalina y otra de Las Higueras, estableciéndose un cronograma de visitas.

En una quinta etapa, se procedió a conformar los grupos de estudiantes que realizarían las visitas. Se realizaron reuniones con cada uno de ellos a los fines de que pudiesen evacuar dudas teóricas pero también referidas a la forma de la exposición. Se elaboró una presentación única en Power Point a los fines de aunar criterios y se realizó la selección de la escuela en la que cada uno participaría teniendo en cuenta la disponibilidad de los mismos por los requerimientos de cursado. Ello requirió en algunos casos tener que pedir autorización a docentes a los fines de que permitieran a los estudiantes realizar las prácticas cuando las mismas coincidían con el horario de cursado.

En la sexta etapa, se realizaron las cuatro visitas a las escuelas. Las exposiciones se estructuraron para durar no más de 60 minutos, dentro de los cuales, en una primera parte los estudiantes explicaban a los alumnos del nivel medio cuáles eran los derechos de las República Argentina sobre los archipiélagos australes -con el debido acompañamiento de los docentes de las cátedras-y en una segunda parte los veteranos de la agrupación daban a conocer sobre su experiencia en la guerra. En esta parte se buscaba generar en los estudiantes y docentes de las escuelas preguntas puntuales sobre las vivencias, siempre buscando recalcar los aspectos negativos de la guerra, pero a la vez rescatando los actos de heroísmo que muchas veces son desconocidos por la sociedad argentina. Concluida la clase se mantuvo una charla con docentes y autoridades de las escuelas a los fines de conocer un poco más sus necesidades a la hora de abordar la temática, pensando en poder seguir trabajando con ellos en lo sucesivo pero también permitiéndonos realizar un relevamiento sobre el tratamiento que le daban al tema.

Como última etapa, se pensó una reunión entre todos los docentes, estudiantes intervinientes y miembros de la Agrupación de Veteranos de Guerra a los fines de intercambiar opiniones que permitan enriquecer las futuras intervenciones. Al mismo tiempo, el encuentro nos permitió consolidar un grupo de trabajo con los 
estudiantes participantes, quienes manifestaron querer seguir participando de este tipo de actividades, incluso colaborando con los docentes en el desarrollo de las futuras prácticas socio-comunitarias a realizarse.

* Evaluación de la experiencia

a. Aportes a la formación de los estudiantes

Una realidad a destacar de la ejecución de las prácticas es sin duda en el nivel de conocimientos adquiridos por lo estudiantes en todo el proceso. Ello en razón de que, si bien los estudiantes abordaron los contenidos en las diferentes materias, lo cierto es que las capacitaciones brindadas, sumado al hecho de tener que armar las clases y, por sobre todas las cosas, interactuar con los estudiantes, autoridades escolares y los propios veteranos, generó en ellos un nivel de conciencia sobre la importancia de la complejidad del tema y el nivel de desconocimiento existente, además de un compromiso con la causa que redundó en que casi todos ellos se ofrecieran a continuar profundizando sobre la temática.

Si bien la participación en la actividad les genera a los estudiantes un antecedente importante, muchos de ellos han decidido seguir colaborando con las actividades, aunque las mismas no les redunden en beneficio alguno. Es así que para principios de 2020 se sumaron a las actividades del Área Malvinas del centro de estudios en miras de ampliar sus conocimientos sobre la cuestión Malvinas, además de participar en todas las actividades de extensión -incluidas las prácticas socio-comunitarias- que estén siendo organizadas.

b. Modificaciones a las prácticas de enseñanza

El desarrollo de las prácticas socio-comunitarias también tuvo su importancia a la hora de la reflexión sobre la forma en que se imparten los contenidos relacionados con los derechos argentinos sobre los archipiélagos australes y sus áreas marítimas circundantes. Empezando por la propia universidad, donde los contenidos que abordan las temáticas son muchas veces escasos y están desconectados entre sí, lo redunda en que los estudiantes no alcancen a conocer en detalle cuales son los lineamientos sobre los que se apoya el reclamo argentino. En lo que respecta a las escuelas de nivel medio en las que nos tocó realizar las prácticas se pudo observar que las docentes a cargo de impartir los contenidos vinculados con los derechos argentinos sobre las islas se encuentran sobrepasadas en lo que al dictado de contenidos se refiere. A ello hay que sumarle las propias limitaciones institucionales o las que se derivan de la condición socio-económica de los estudiantes que acuden a las escuelas.

De las charlas que se tuvieron al finalizar la ejecución de las prácticas y fruto del diagnóstico antes mencionado, se pensó en armar capacitaciones para los docentes 
del nivel medio. La propuesta realizada en ese sentido a las docentes y autoridades de las escuelas visitadas recibió gran aceptación.

En lo que hace al ámbito universitario, se pensó en generar una cátedra abierta sobre la cuestión Malvinas a las que se puedan sumar todos los estudiantes de las carreras de la Facultad de Ciencias Humanas que tengan algún interés en la temática. Dicha cátedra sería organizada por los docentes que forman el centro de estudios y tendría como finalidad analizar la cuestión Malvinas desde una mirada interdisciplinaria y enfocada también a formar a los estudiantes y docentes que estén dispuestos a llevar adelante actividades de extensión sobre la temática.

c. Adecuación del proyecto a las necesidades de la comunidad

Sin lugar a dudas, esta primera experiencia de práctica socio-comunitaria nos permitió ahondar más profundamente sobre las necesidades existentes en la comunidad educativa a la hora de hablar de los derechos argentinos sobre las islas. Si bien el Ateneo de Estudios Internacionales (AEI) viene trabajando sobre la temática desde el año 2012, lo que nos ha permitido conocer de esas limitaciones en diferentes centros de ex combatientes, es la primera vez que podemos hacer un trabajo de campo en escuelas de nivel medio de la ciudad.

De esta manera, pudimos corroborar el nivel de conocimientos de docentes y estudiantes de las escuelas de nivel medio y que gracias a las exposiciones dadas junto a los veteranos de guerra de la agrupación nos permitió hacer un aporte importante para cubrir las necesidades de las mencionadas instituciones. Ello lo pudimos corroborar a partir de las charlas posteriores con las autoridades de las escuelas y el pedido para que sigamos trabajando con ellos todos los años.

d. Aportes a la comunidad

Consideramos que las prácticas han permitido, a la par de poner los conocimientos sobre la temática a disposición de las escuelas de nivel medio del Gran Río Cuarto, generar una red de contactos tanto dentro de la universidad como así también entre las escuelas, que nos permite pensar en comenzar a trabajar a mediano y largo plazo a los fines de brindar capacitaciones permanentes a los docentes de las escuelas de nivel medio. Ello al margen de las diferentes actividades de extensión que se puedan estar realizando.

Por otro lado, la ejecución de las mismas ha permitido generar una vinculación entre la Universidad y la Agrupación de Veteranos que no existió nunca desde 1982. Durante todo el proceso los veteranos que intervinieron han podido intercambiar opiniones con autoridades de nuestra casa de altos estudios, como así también con docentes y alumnos de las diferentes carreras que intervinieron en la práctica. Ello ha redundado también en una mayor vinculación de la comunidad universitaria en los acontecimientos de la guerra y las diferentes aristas que rodean a la cuestión 
Malvinas. Por ello podríamos concluir que el beneficio fue múltiple, para todos los actores intervinientes y de ida y vuelta.

e. Grado de participación de los actores intervinientes en el proyecto

El compromiso de los actores participantes fue muy alto. Empezando por los docentes intervinientes, tanto los universitarios como los de las escuelas. Los primeros, por el armado y ejecución del proyecto de práctica, que supone un esfuerzo adicional que hay que sumar a las tareas cotidianas que los docentes deben llevar adelante. En este caso, no solo convocando a los estudiantes, capacitándolos, sino también en lo que respecta a la ejecución de las prácticas, lo que requiere toda una logística de coordinación con los estudiantes y las autoridades y docentes de las escuelas.

En lo que respecta a los docentes de las escuelas de nivel medio visitadas, tanto ellas como las autoridades se mostraron interesadas en el tema desde el principio, generando de ese modo los espacios para que las exposiciones se pudiesen llevar adelante. En ese sentido, en varias de las escuelas se reunió a los estudiantes de varios cursos para que la mayor cantidad de estudiantes pudiesen tomar aprender y en algunos casos, muchos docentes, de diferentes asignaturas se sumaron a las charlas, siempre intentando incentivar a la participación de sus estudiantes.

En relación a los estudiantes de las escuelas, las respuestas fueron variadas. En algunas los estudiantes participaron más que otras. Ello dependió mucho del incentivo generado por los profesores, previo al encuentro con los estudiantes de nuestra universidad. En algunos casos, el hecho de haber entre los estudiantes de las escuelas familiares de veteranos ayudó mucho a generar interesantes debates.

Finalmente, los veteranos de guerra que participaron mostraron una gran colaboración tanto antes, como durante la ejecución de las prácticas. Desde el principio nunca tuvieron excusas a la hora de concretar reuniones a los fines de ir definiendo los cursos de acción a seguir. Lo mismo a la hora de tener que reunirse con los estudiantes que ejecutarían las prácticas. A la hora de las visitas a las escuelas se mostraron predispuestos a dar a conocer sus experiencias en la guerra frente a las preguntas de los estudiantes. Además se ofrecieron a seguir trabajando en actividades de este tipo a futuro.

\section{f. Posibilidades de sustentabilidad del proyecto}

Entre los obstáculos que se pueden mencionar a la hora de llevar adelante las prácticas, podemos mencionar la gran cantidad de actividades que la ejecución de la PSC demanda, sobre todo a la hora de convocar a los estudiantes, armar los grupos de trabajo, capacitarlos a lo que hay que sumar toda la logística necesaria para poder coordinar las actividades con las instituciones educativas de nivel medio y los estudiantes que concurrirán a dar las charlas. Todas estas actividades fueron 
desarrolladas por un pequeño grupo de docentes -algunos con dedicación simple- e integrantes del Ateneo de Estudios Internacionales (AEI), lo que sin dudas requirió de un gran esfuerzo.

Sin embargo, en relación a las posibilidades de continuar con la experiencia, no podemos dejar de considerar que las demandas existen y seguirán existiendo, los docentes estamos ahora más formados y capacitados para conformar los grupos y los vínculos con las instituciones son más que sólidos, por lo que se considera factible poder seguir con este tipo de actividades.

\section{Conclusión}

A modo de cierre podríamos decir que los proyectos de prácticas socio-comunitarias implementadas por la Universidad Nacional de Río Cuarto, ha demostrado con el correr de los años ser de gran utilidad a la hora de permitir el vínculo entre la comunidad universitaria y la sociedad toda. La idea de incorporar en el corto o mediano plazo a las mismas en los planes de estudio de las carreras que se dictan en la universidad no hará más que fortalecer ese vínculo y permitirá a los estudiantes que participan de ellas, adquirir conciencia sobre la importancia de brindar sus conocimientos a la comunidad.

Concretamente, la ejecución de la práctica "Malvinas en las escuelas. La construcción de la cuestión Malvinas en el sistema educativo de nivel medio en el Gran Río Cuarto” nos ha servido para conocer cuán importante puede ser una actividad de extensión a la hora de satisfacer las necesidades educativas de las instituciones académicas del nivel medio de nuestra ciudad. Sin embargo, también ha permitido a los miembros de la comunidad universitaria redefinir el rol que la universidad está llamada a cumplir y lo importantes que es para la sociedad la participación en este tipo de iniciativas.

Para quienes trabajamos cotidianamente, sea investigando o enseñando sobre la cuestión Malvinas, este tipo de prácticas nos sirven a los fine de generar una mayor "malvinización" en la sociedad argentina, termino al que debemos entender no como una reivindicación de la intervención militar en las decisiones políticas que corresponden a la sociedad civil -dentro de un régimen democrático- sino a la reconstrucción desde los diferentes sectores que la componen, para que se construyan políticas de Estado que hagan posible su recuperación de manera legítima y pacífica (García, 2019).

\section{Referencias bibliográficas}


Consejo Social Universitario. (2019). UNRC - Secretaría de Planeamiento y Relaciones Institucionales. Recuperado el 2020 de abril de 28, de https://www.unrc.edu.ar/unrc/planeamiento/docs/psc/2019/infconsejosocial_Ampliaci\%C3\%B3n\%202019.pdf

García, G. E. (16 de Enero de 2019). Observatorio Malvinas. Recuperado el 28 de Abril de 2020, de https://observatoriomalvinassite.wordpress.com/2019/01/16/elcapitan-castagnari-perspectivas-y-desafios-para-la-sociedad-argentina/

UNRC. (2020). Secretaria de Planeamiento y Relaciones Institucionales. Recuperado el 28 de abril de 2020, de https://www.unrc.edu.ar/unrc/planeamiento/psc.php 